\title{
Estudo retrospectivo da prevalência de fenômenos de retenção e extravasamento de muco - correlação clínico-histopatológica
}

\author{
Retrospective study on the prevalence of mucus retention and \\ extravasation phenomena - clinical and histopatological correlation
}

Flávia Godinho Costa Wanderley*

Lívia Prates Soares Zerbinati**

Sílvia Regina de Almeida Reis ${ }^{* * *}$

Alena Ribeiro Alves Peixoto Medrado****

\section{Resumo}

Objetivo: mucocele de retenção, mucocele de extravasamento e rânula são lesões descritas na literatura como sendo decorrentes de trauma mecânico no ducto secretório das glândulas salivares. Entretanto, muitos pacientes que apresentam essas lesões não relatam a associação do diagnóstico histopatológico, obtido por meio da biópsia, com a história clínica de traumatismo prévio. Sendo assim, a finalidade desta pesquisa foi estabelecer a prevalência de mucocele de retenção, mucocele de extravasamento e rânula e coletar dados clínicos dos pacientes, a fim de confirmar a história de trauma associado a essas lesões. Métodos: neste estudo observacional retrospectivo, todos os casos de mucocele de retenção, mucocele de extravasamento e rânula foram recuperados dos arquivos do Serviço de Patologia Bucal de uma Instituição de Ensino Superior privada, abrangendo o período de 2001 a 2012. Resultados: observou-se maior prevalência das lesões em indivíduos do sexo feminino $(50,7 \%)$, com faixa etária predominante situada na primeira década de vida (53,6\%), sem significância estatística. O diagnóstico histopatológico mais prevalente foi o de mucocele de extravasamento $(85,9 \%)$, corroborando a hipótese diagnóstica inicial para essa patologia $(77,5 \%)(p<0,05)$. O lábio inferior foi o local de maior acometimento dessas lesões $(60,6 \%)(p<0,05)$. A maior parte dos relatos colhidos na anamnese não indicou ocorrência significativa de história prévia de trauma percebida pelos pacientes $(66,2 \%)$. Conclusão: frente à significativa casuística dos fenômenos de retenção e extravasamento de muco observada no presente estudo, urge a necessidade de se ampliar os dados da anamnese, a fim de que a associação do trauma junto a essas lesões seja devidamente documentada.

Palavras-chave: Mucocele. Rânula. Trauma.

\section{Introdução}

Mucoceles são lesões nodulares flutuantes, benignas, de coloração translúcida azulada ou da mesma tonalidade da mucosa. Geralmente são autolimitadas e assintomáticas e estão relacionadas com as glândulas salivares menores, sendo uma ocorrência rara o acometimento das glândulas salivares maiores ${ }^{1}$.

Estudante de Iniciação Científica da Escola Bahiana de Medicina e Saúde Pública, Salvador, Bahia, Brasil. Bolsista Fapesb e integrante voluntária do Programa de Educação Tutorial PET-Odonto-BAHIANA.

Doutora em Cirurgia e Traumatologia Bucomaxilofacial pela PUCRS, Porto Alegre, Rio Grande do Sul, Brasil.

Doutora em Patologia Oral pela Universidade Livre de Berlim. Professora adjunta do curso de Odontologia da Escola Bahiana de Medicina e Saúde Pública, Salvador, Bahia, Brasil.

Doutora em Patologia Humana pela Fiocruz/UFBA, professora adjunta da Escola Bahiana de Medicina e Saúde Pública, Salvador, Bahia, Brasil. 
Preferencialmente localizada no lábio inferior, a mucocele desenvolve-se mais raramente no lábio superior, palato, região retromolar, mucosa bucal, freio lingual e dorso da língua ${ }^{2}$. Acomete mais o sexo feminino e indivíduos leucodermas. A faixa etária mais acometida engloba indivíduos na segunda e terceira décadas de vida ${ }^{3}$.

Acredita-se que a mucocele seja resultante de um trauma mecânico no ducto secretório das glândulas salivares, causando ruptura ou obstrução do ducto. A ruptura do ducto libera mucina para o estroma de tecido conjuntivo, ao passo que a obstrução do canal retém muco no interior do ducto e/ou ácino. A retenção de muco é menos frequente que o extravasamento. Além disso, o extravasamento de muco é encontrado principalmente em crianças e adultos jovens, enquanto o fenômeno de retenção se desenvolve predominantemente em idosos ${ }^{4}$.

Histologicamente, a mucocele de extravasamento é caracterizada por uma parede cística desprovida de epitélio que é delimitada por tecido de granulação e por células inflamatórias. Há depósitos mucinosos no lúmen do cisto ${ }^{5}$. Na mucocele de retenção, os ductos glandulares apresentam-se dilatados e a mucina frequentemente encontra-se no interior do lúmen de tais estruturas. O tratamento das mucoceles envolve a excisão cirúrgica da lesão e dissecção cuidadosa das glândulas salivares menores adjacentes afetadas, a fim de evitar recidiva ${ }^{6}$.

Por sua vez, a rânula, uma mucocele que aparece no assoalho da boca, está geralmente relacionada com os sistemas de ductos das glândulas sublinguais e, menos frequentemente, com a glândula submandibular ${ }^{7}$. É maior que a mucocele $(>2 \mathrm{~cm})$, e o tratamento preconizado inclui a remoção cirúrgica da glândula sublingual e/ou marsupialização da lesão ${ }^{8}$.

O objetivo deste estudo foi estabelecer a prevalência de mucocele de retenção, mucocele de extravasamento e rânula e coletar dados clínicos dos pacientes, a fim de confirmar a história de trauma associado a essas lesões.

\section{Materiais e método}

O presente estudo foi submetido à avaliação do Comitê de Ética e Pesquisa em Humanos, sendo aprovado pelo protocolo $\mathrm{n}^{\mathrm{0}}$ 12511713.1.0000.5544.

Neste estudo observacional retrospectivo, todos os casos de mucocele de retenção, mucocele de extravasamento e rânula foram recuperados dos arquivos do Serviço de Patologia Bucal da Escola Bahiana de Medicina e Saúde Pública, Salvador, Bahia, Brasil, abrangendo o período de 2001 a 2012.

Os casos de mucocele de retenção, mucocele de extravasamento e rânula foram selecionados a partir da avaliação dos prontuários. Tendo como base o diagnóstico histopatológico de cada caso, foram avaliadas a frequência e a distribuição geral das amostras quanto a sexo, idade, localização da lesão e histórico de trauma. De acordo com os critérios de inclusão no estudo, foram utilizados apenas os prontuários diagnosticados com as lesões já descritas. O critério de exclusão adotado para este estudo pautou-se na eliminação de todos os casos não totalmente completos com dados clínicos.

Os dados foram tabulados em planilha do Excel (Windows 7), sendo realizada uma análise descritiva com percentual dos índices relativos às lesões. Após a tabulação dos dados, foi realizada análise estatística através do teste exato de Fisher para determinar o grau de significância estatística dos resultados. As diferenças foram consideradas estatisticamente significativas quando o valor de $p<0,05$.

\section{Resultados}

Do total de 71 prontuários analisados, verificou-se que $35(49,3 \%)$ corresponderam a indivíduos do sexo masculino e $36(50,7 \%)$ do sexo feminino (Tab. 1). Em relação à distribuição etária dos indivíduos analisados, esta variou entre a primeira e a quinta década de vida. O maior percentual de lesões de mucocele de retenção, mucocele de extravasamento e rânula concentrou-se na primeira e segunda décadas de vida (Tab. 2).

Tabela 1 - Gênero dos indivíduos portadores de mucocele de retenção, de extravasamento e rânula

\begin{tabular}{l|c|c}
\hline \multicolumn{1}{c|}{ Sexo } & $\mathrm{n}$ & $\%$ \\
\hline Masculino & 35 & 49,3 \\
Feminino & 36 & 50,7 \\
Total & 71 & 100
\end{tabular}

Teste exato de Fisher $(p>0,05)$

Tabela 2 - Faixa etária dos indivíduos portadores de mucocele de retenção, de extravasamento e rânula

\begin{tabular}{l|c|c}
\hline \multicolumn{1}{|c|}{ Idade } & $\mathrm{n}$ & $(\%)$ \\
\hline 1 $^{\text {a }}$ década de vida & 38 & 53,6 \\
$2^{2}$ a década de vida & 22 & 31,0 \\
$3^{3}$ década de vida & 03 & 4,2 \\
$4^{\text {a }}$ década de vida & 03 & 4,2 \\
$5^{\text {a }}$ década de vida & 05 & 7,0 \\
Total & 71 & 100 \\
\hline
\end{tabular}

Teste exato de Fisher $(p>0,05)$

Os diagnósticos histopatológicos, descritos nos laudos das biópsias, anexados aos seus respectivos prontuários, evidenciaram maior prevalência de mucocele de extravasamento $(85,9 \%)$ e mucocele de retenção de muco $(9,9 \%)$, seguidos de rânula $(4,2 \%)$ (Tab. 3 ). 
Tabela 3 - Diagnóstico histopatológico das lesões, segundo os prontuários analisados

\begin{tabular}{l|c|c}
\hline \multicolumn{1}{c|}{ Diagnóstico histopatológico } & $\mathrm{n}$ & $(\%)$ \\
\hline Mucocele de extravasamento & 61 & 85,9 \\
Mucocele de retenção & 07 & 9,9 \\
Rânula & 03 & 4,2 \\
Total & 71 & 100 \\
\hline
\end{tabular}

A Tabela 4 representa a suspeita diagnóstica feita pelos cirurgiões-dentistas e encontradas nos prontuários analisados. Sendo assim, dos 71 prontuários, $55(77,5 \%)$ possuíam a hipótese diagnóstica de mucocele, $8(11,3 \%)$ de rânula, $4(5,6 \%)$ de fibroma traumático, $2(2,8 \%)$ de granuloma piogênico, 1 de hiperplasia inflamatória $(1,4 \%)$ e $1(1,4 \%)$ de neuroma.

Tabela 4 - Hipótese diagnóstica, segundo os prontuários analisados

\begin{tabular}{l|c|c}
\hline \multicolumn{1}{c|}{ Suspeita diagnóstica } & $\mathrm{n}$ & $(\%)$ \\
\hline Mucocele ${ }^{*}$ & 55 & 77,5 \\
Rânula & 08 & 11,3 \\
Fibroma traumático & 04 & 5,6 \\
Granuloma piogênico & 02 & 2,8 \\
Hiperplasia inflamatória & 01 & 1,4 \\
Neuroma & 01 & 1,4 \\
Total & 71 & 100 \\
\hline Teste exato de Fisher $(p<0,05)$ &
\end{tabular}

Teste exato de Fisher $(p<0,05)$

A localização da lesão, segundo os prontuários analisados, está retratada na Tabela 5. Do total de lesões apresentadas, $60,6 \%$ localizaram-se no lábio inferior, $9,9 \%$ no assoalho da boca e na mucosa jugal, $8,5 \%$ no ventre da língua, $4,2 \%$ no palato, $2,8 \%$ no dorso da língua e 1,4\% no lábio superior e nas regiões sublingual e submandibular.

Tabela 5 - Localização da lesão, segundo os prontuários analisados

\begin{tabular}{l|c|c}
\hline \multicolumn{1}{c|}{ Local } & $\mathrm{n}$ & $(\%)$ \\
\hline Lábio inferior ${ }^{*}$ & 43 & 60,6 \\
Assoalho da boca & 07 & 9,9 \\
Mucosa jugal & 07 & 9,9 \\
Ventre da língua & 06 & 8,5 \\
Palato & 03 & 4,2 \\
Dorso da língua & 02 & 2,8 \\
Lábio superior & 01 & 1,4 \\
Região sublingual & 01 & 1,4 \\
Região submandibular & 01 & 1,4 \\
Total & 71 & 100 \\
\hline Teste exato de Fisher $(p<0,05)$. &
\end{tabular}

A Tabela 6 mostra o histórico de trauma referido nos prontuários. Do total de 71 prontuários, 47 $(66,2 \%)$ não relataram história de trauma pelos pacientes e $24(33,8 \%)$ referiram origem traumática.

Tabela 6 - Histórico de trauma referido nos prontuários

\begin{tabular}{l|c|c}
\hline \multicolumn{1}{c|}{ Histórico de trauma } & $\mathrm{n}$ & $(\%)$ \\
\hline Não referido & 47 & 66,2 \\
Origem traumática & 24 & 33,8 \\
Total & 71 & 100 \\
\hline
\end{tabular}

Teste exato de Fisher $(p>0,05)$.

\section{Discussão}

O presente estudo procurou estabelecer a prevalência de mucocele de retenção, mucocele de extravasamento e rânula, coletando dados clínicos dos pacientes, a fim de confirmar a história de trauma associado a essas lesões.

Dos 71 prontuários analisados, entre os anos de 2001 e 2012, $36(50,7 \%)$ corresponderam ao sexo feminino, enquanto $35(49,3 \%)$ representaram o sexo masculino, sendo essa diferença estatisticamente significativa. Hayashida et al. ${ }^{2}$ (2010) relataram resultados iguais aos do presente estudo ao analisarem uma amostra de 173 pacientes. Esses autores observaram uma maior prevalência das lesões no sexo feminino. No entanto, não há na literatura um consenso a respeito da ocorrência dessas lesões em relação ao gênero, embora outros ratifiquem uma maior frequência em indivíduos do sexo masculino $0^{9-11}$.

Tem sido relatado um maior número de casos de fenômenos de retenção e extravasamento de muco em pacientes jovens, com faixa etária situada entre a segunda e a terceira década de vida ${ }^{12}$. Possivelmente, essa maior prevalência pode ser justificada por episódios recorrentes de trauma nesses indivíduos. $\mathrm{O}$ presente estudo contrastou esses aspectos citados na literatura, evidenciando que tais lesões ocorrem mais na primeira e segunda décadas de vida.

Do total de lesões apresentadas, $60,6 \%$ localizaram-se no lábio inferior, 9,9\% no assoalho da boca, $9,9 \%$ na mucosa julgal, $8,5 \%$ no ventre da língua, $4,2 \%$ no palato, $2,8 \%$ no dorso da língua e $1,4 \%$ no lábio superior e nas regiões sublingual e submandibular ( $p<0,05)$. Hayashida et al. ${ }^{2}(2010)$, ao analisarem 173 casos, verificaram que o lábio inferior (78\% dos casos) também foi o local de maior prevalência de fenômenos de retenção e extravasamento de muco, coincidindo, então, esse resultado com o do presente estudo.

Os fenômenos de retenção e extravasamento de muco são muito frequentes em meio aos diagnósticos histopatológicos, usualmente estabelecidos pelos diferentes Serviços de Patologia. Em um estudo realizado 
por Chen et al. ${ }^{11}$ (2010), no qual foram analisados 2.762 casos, o diagnóstico histopatológico mais frequente foi de mucocele (955 casos, $34,5 \%$ ), seguido por úlcera traumática (818 casos, 29,6\%). Em outro estudo, desenvolvido por Neto et al. ${ }^{13}$ (2012), observouse que o diagnóstico histopatológico mais frequente foi de hiperplasia fibroepitelial $(48,53 \%)$, seguido de mucocele $(48,10 \%)$. Em nosso estudo, restringimos os laudos do Serviço de Histopatologia a três categorias: mucocele de retenção, mucocele de extravasamento e rânula. Nesse contexto, observou-se uma significativa prevalência de mucocele $(95,8 \%)$.

Considerando, ainda, os dados levantados, tem-se que, das suspeitas diagnósticas formuladas pelos cirurgiões-dentistas, $88,85 \%$ foram posteriormente confirmadas pelos diagnósticos histopatológicos e $11,15 \%$ tiveram resultados diversos. Se comparados aos resultados apresentados por Neto et al. ${ }^{13}$ (2012), das suspeitas diagnósticas formuladas pelos cirurgiões-dentistas, $59,82 \%$ foram posteriormente confirmadas pelos diagnósticos histopatológicos e $40,18 \%$ tiveram resultados diversos.

Segundo Gupta e Kaejodkar ${ }^{8}$ (2011), os fenômenos de retenção e extravasamento de muco são ocasionados por trauma mecânico no ducto secretório das glândulas salivares. Essa afirmativa não pode ser confirmada pelos achados da presente pesquisa, visto que, de 71 prontuários, $47(66,2 \%)$ não referiram trauma pelos pacientes e $24(33,8 \%)$ referiram origem traumática. Esses resultados, porém, ratificam os achados de García et al. ${ }^{12}$ (2009), os quais estudaram 68 amostras e apenas um terço dos pacientes relatou trauma anterior, com a maior parte dos casos sem fator etiológico evidente. Além disso, o presente estudo corrobora os resultados apresentados por Chen et al. ${ }^{11}$ (2010), em que, das 2.788 amostras analisadas, apenas $10 \%$ forneciam história confirmada de trauma.

Esses diferentes resultados refletem que, embora o trauma seja, de fato, um fator determinante para o desenvolvimento dessas lesões, a sua correlação com o estabelecimento da lesão requer um resgate fiel do seu histórico por parte dos pacientes. Muitas vezes, os dados coletados durante a anamnese são insuficientes e não esclarecedores.

Cabe ao cirurgião-dentista a responsabilidade primária de registrar na ficha clínica, com a maior riqueza de detalhes possível, todos os aspectos relevantes capazes de contribuir para o desenvolvimento de quaisquer lesões do complexo bucomaxilofacial, em especial o grupo de lesões que foi objeto desta investigação.

\section{Conclusão}

Devido à dificuldade em se verificar a percepção do trauma por parte dos portadores dos fenômenos de retenção e extravasamento de muco, sugere-se que o profissional de saúde realize uma acurada anamnese, com o objetivo de buscar o correto diagnóstico da patologia apresentada pelo paciente. Dessa forma, fica evidente que, quanto mais minuciosa for a anamnese, mais preciso será o diagnóstico e, portanto, mais elucidativa será a etiologia da lesão. Tal fato contribuirá para a instituição de um tratamento mais adequado para a patologia em questão.

\section{Abstract}

Objective: retention mucocele, extravasation mucocele, and ranula are lesions described in literature as being derived from mechanical trauma in salivary gland secretory duct. However, many patients with these lesions did not report the association of histopatological diagnosis obtained by biopsy to a history of previous clinical trauma. Thus, the purpose of this research was to establish the prevalence of retention and extravasation mucocele, and ranula, and collect data from clinical patients in order to confirm the history of trauma associated to these lesions. Methods: in this retrospective observational study all cases of retention mucocele, extravasation mucocele, and ranula were retrieved from the files of the Department of Oral Pathology of a private Dentistry School, from 2001 to 2012. Results: this study presented a higher prevalence of lesions in female individuals $(50.7 \%)$, predominantly in the 1 st decade of life (53.6\%) regarding age group, and with no statistical significance. The most prevalent histopatological diagnosis was extravasation mucocele (85.9\%), confirming the initial diagnostic hypothesis for this pathology (77.5\%) ( $p<0.05)$. The lower lip was the site most affected by these lesions (66.2\%) ( $p<0.05)$. Most reports collected in anamnesis indicated no significant occurrence of history of previous trauma perceived by patients (66.2\%). Conclusions: due to the significant occurrence of mucus retention and extravasation phenomena observed in the present study there is an urgent need to enhance anamnesis data so that the association of trauma to these lesions is better documented.

Keywords: Mucocele. Ranula. Trauma.

\section{Referências}

1. Rangeeth BN, Moses J, Reddy VKK. A rare presentation of mucocele and irritation fibroma of the lower lip. Contemporary Clinical Dentistry 2010; 1(2):111-4.

2. Hayashida AM, Zerbinatti DCZ, Balducci I, Cabral LAG, JD Almeida. Mucus extravasation and retention phenomena: a 24-year study. Bio Med Central Oral Health 2010; 10-15.

3. Kim JH, Park HY, Hong SP, Ahn SK. Concurrent occurrence of mucocele and pyogenic granulom. Ann Dermatol 2011; 23(1):108-10.

4. Arunachalam P, Priyadharshini N. Recurrent plunging ranula. J Indian Assoc Ped Surg 2010; 15(1):36-8.

5. Choi HJ, Kim SG, Kim JD, Kim JH, Kim JH, Kim SM. A case of bilateral submandibular gland mucoceles in a 16-month-old child. Korean J Ped 2012; 55(6):215-8. 
6. Seo J, Bruno I, Artico G, Vechio AD, Migliari DA. Oral mucocele of unusual size on the buccal mucosa: clinical presentation and surgical approach. The Open Dent J 2012; 6:67-8.

7. Dietrich EM, Vasilios B, Maria L, Styliani P, Konstantinos A. Sublingual-plunging ranula as a complication of supra omohyoid neck dissection. Int J Surg Case Reports 2011; 2(6):90-2.

8. Gupta A, Karjodkar FR. Plunging ranula: a case report. ISRN Dent 2011; 2011:1-5.

9. Walvekar RR, Peters G, Hardy E, Alsfeld L, Stromeyer FW, Anderson D, et al. Robotic-assisted transoral removal of a bilateral floor of mouth ranulas. World J Surg Oncol 2011; 9(78).

10. Lee E, Cho SH, Park CJ. Clinical and immunohistochemical characteristics of mucoceles. Ann Dermatol 2009; 21(4):34551 .

11. Chen JY, Wang WC, Chen YK, Lin LM. A retrospective study of trauma-associated oral and maxillofacial lesions in a population from southern Taiwan. J Applied Oral Science 2010; 18(1):5-9.

12. García JY, España-Tost AJ, Berini-Aytés L, Gay-Escoda C. Treatment of oral mucocele - scalpel versus C02 laser. Med Oral Patol Oral Cir Bucal 2009; 14 (9):469-74.

13. Neto BD, Medrado AP, Reis SRA. Levantamento epidemiológico dos diagnósticos histopatológicos de um Centro de Referência em Patologia Bucomaxilofacial em um período de 10 anos. Rev Baiana Odontol 2012; 3(1):3-15.

Endereço para correspondência:

Alena Ribeiro Peixoto Medrado

Rua Rodolfo Coelho Cavalcante 90/502,

Jardim Armação

41.750.166 Salvador-BA

Fones: (71) 32722042 - 88320218

E-mail: apmedrado@bahiana.edu.br

Recebido: 25/09/2013. Aceito: 04/01/2014. 